\title{
PROJETO EXERCÍCIO E CORAÇÃO: UMA DÉCADA A SERVIÇO DA COMUNIDADE
}

\author{
PROJECT EXERCISE AND HEART: \\ SERVING THE COMMUNITY FOR A DECADE \\ *Cláudia Lúcia de Moraes Forjaz, **Bruno Temoteo Modesto, ***Teresa Bartholomeu, \\ ****Luiz Augusto Riani Costa, *****Taís Tinucci
}

\section{RESUMO}

O sedentarismo é um fator de risco para as doenças crônicas e, portanto, a prática regular de atividades físicas auxilia na prevenção e combate dessas doenças. Diversas campanhas públicas têm incentivado as pessoas a praticarem exercícios em locais públicos. No entanto, a prática muitas vezes é executada sem a orientação de um profissional, o que pode representar um risco à saúde, principalmente para pessoas com doenças cardiovasculares. Diante dessa problemática, há dez anos, o Projeto Exercício e Coração foi criado para orientar/prescrever a prática segura de atividade física para frequentadores de locais públicos que se exercitam sem supervisão. O projeto atua de forma contínua no parque Fernando Costa e no Hospital Universitário da USP, além de participar de campanhas públicas de saúde. Nesses dez anos, o projeto avaliou mais de I.43I pessoas no parque, ministrou mais de 2.IO9 aulas de alongamento, participou de mais de 28 eventos, treinou mais de I5O monitores e publicou quarenta resumos e nove artigos científicos completos relacionados aos seus dados. Assim, o Projeto Exercício e Coração é uma atividade de extensão que tem a preocupação de levar à comunidade os conhecimentos acadêmicos produzidos pela universidade, integrando pesquisa, ensino e extensão.

Palavras-chave: Atividade física não supervisionada. Risco cardiovascular. Parques públicos.

\section{ABSTRACT}

Sedentary lifestyle is a risk factor for chronic disease development, and consequently regular physical activity can help to prevent and control these diseases. Thus, many public campaigns have been created to motivate people to become active, and many subjects are practicing exercises in public facilities. However, at these sites, physical practice is frequently performed without any professional advice, which increases its cardiovascular risks especially in people with cardiovascular diseases. To deal with this problem, the Exercise and Heart Project was created IO years ago to prescribe secure physical activity to people who exercise in public facilities without supervision. The project has continuous activities in Fernando Costa park and in the USP University Hospital, and it also participates at public health campaigns. Throughout the years, the project evaluated more than I,43I persons in the park, gave more than 2,IO9 stretching class, participated in more than 28 health events, trained more than 150 monitors, and published forty abstracts and nine complete scientific articles based on its data. In conclusion, the Exercise and Heart Project is a community extension activity of the university that takes the academic knowledge to the community, and also integrates extension, teaching and research.

Key words: Unsupervised physical activity. Cardiovascular risk. Public facilities.

\footnotetext{
* Professora doutora e livre docente do Laboratório de Hemodinâmica da Atividade Motora (LAHAM) da Escola de Educação Física e Esporte da Universidade de São Paulo (EEFE-USP) - Av. Prof. Mello Moraes, 65 - 05508-030 - Cidade Universitária São Paulo - SP - e-mail: cforjaz@usp.br. ** Bacharel em Esporte pela EEFE-USP. **** Professora especialista da EEFE-USP. ***** Médico especialista da EEFE-USP. ***** Professora doutora e docente da EEFE-USP.
} 


\section{INTRODUÇÃO}

O combate às doenças crônicas não transmissíveis (DCNT) é uma preocupação mundial. Das 57 milhões de mortes no mundo em 2008, 36 milhões ocorreram em razão dessas doenças. No Brasil, elas são responsáveis por $72 \%$ das mortes, com destaque para as doenças do aparelho circulatório (3I,3\%) [4].

As DCNT são, em sua maioria, causadas por fatores de risco ligados às modificações nos hábitos de vida, principalmente às dietas calóricas e ricas em sal e gorduras e ao aumento expressivo do sedentarismo. De fato, o número de sedentários tem aumentado no Brasil. Em 2006, I3,2\% dos entrevistados num inquérito populacional relataram não praticar nenhuma atividade física e, em 2008, este índice foi de I7,4\% [5].

É sabido que pessoas sedentárias têm risco entre $20 \%$ e $30 \%$ maior de mortalidade por todas as causas e, principalmente, por doenças cardiovasculares [I8]. A atividade física regular reduz o risco de diabetes, obesidade, dislipidemia, câncer de mama e de cólon, osteoporose [I9] e promove benefícios cardiovasculares já bem estabelecidos [I5,I6]. Desta forma, a atividade física é uma importante estratégia para a prevenção e o controle das DGNT. De fato, o recém-lançado Plano de Ações Estratégicas para o Enfrentamento das DCNT 20II-20I2 no Brasil estabelece o aumento da prática de atividades físicas como um dos principais alicerces das intervenções de saúde [4]. Entretanto, apesar de o plano ser novo, o incentivo à prática de atividade física já ocorre há vários anos em nosso país, por exemplo, com o Programa Agita São Paulo [Io]. Como resultado desse incentivo, muitas pessoas começaram a se exercitar. De fato, o número de sujeitos ativos no estado de São Paulo, onde esse programa se desenvolve, aumentou cerca de $20 \%$ de 2002 a 2008 [II].

Para se tornarem mais ativas, várias pessoas optam por realizar exercícios físicos planejados, como caminhar, correr ou fazer ginástica, em locais públicos, como praças e parques. Porém, nestes locais, a prática é frequentemente realizada sem a orientação de um profissional [8].

Ainda que do ponto de vista populacional os benefícios da prática de exercícios físicos sem supervisão suplantem os possíveis riscos [I5], do ponto de vista individual, a execução de atividades inadequadas pode deflagrar eventos cardiovasculares agudos, como parada cardíaca ou infarto. Dessa forma, para maximizar os benefícios e minimizar os riscos individuais, é importante que os praticantes façam uma avaliação para identificar seu risco $[8,15]$ e recebam uma prescrição ou, no mínimo, uma orientação sobre a prática adequada. Estes procedimentos são especialmente importantes para quem vai se exercitar sem acompanhamento. Diante desse quadro, o Projeto Exercício e Cioração foi criado, visando a estimular e a orientar a prática segura de atividades físicas em locais públicos [9].

O projeto teve início em 2000, no parque Fernando Costa (Água Branca), e continua lá até hoje. Em 2002, o projeto começou a realizar ações pontuais, atuando em outros sete parques de São Paulo; realizando atividades no Projeto Estação USP de 2003 a 2006, e participando de campanhas de saúde pública desde 2006. Além disso, em 2008, as atividades contínuas do projeto se estenderam para o Hospital Universitário da USP (HU-USP).

Por estar na universidade, além do cunho assistencial, o projeto tem envolvimento com o ensino, através do treinamento de alunos que atuam como monitores de suas atividades, e também tem produzido conhecimentos científicos vinculados à sua atuação. No presente artigo pretendemos descrever as atividades desenvolvidas pelo projeto nesses três âmbitos (extensão, ensino e pesquisa), demonstrando os resultados obtidos até o momento.

\section{OBJETIVOS}

No aspecto extensão, o Projeto Exercício e Coração objetiva estimular e dar subsídios para a prática segura de atividades físicas visando à melhora e manutenção da saúde e da qualidade de vida da população. Como objetivos específicos, ele pretende:

- divulgar a importância da prática regular de atividades físicas;

- estimular a população a essa prática;

- levantar o risco individual para essa prática;

- sugerir condutas anteriores à prática, se necessárias;

- fornecer orientação/prescrição individualizada das atividades a serem executadas;

- fornecer subsídios para a execução correta dessas atividades.

No aspecto do ensino, o projeto visa a preparar alunos de graduação e pós-graduação em Educação Física para atuarem como profissionais competentes na área do exercício físico para a saúde, complementando 
os conhecimentos teóricos obtidos em sala de aula e, principalmente, fornecendo-lhes a experiência prática supervisionada para essa atuação.

No âmbito da pesquisa, o projeto tem por objetivo produzir conhecimento aplicado sobre a forma eficaz de propor e executar a prática segura e benéfica de atividade física para populações que se exercitam em locais públicos sem supervisão direta.

\section{MÉTODOS} de atuação:

O projeto possui atualmente três vertentes

- uma contínua, no parque Fernando Costa;

- uma contínua, no Hospital Universitário da USP;

- atuações pontuais junto a outros projetos, eventos ou campanhas de saúde.

Todas as atividades têm embasamento teórico nas Diretrizes do Colégio Americano de Medicina Esportiva (ACSM) [I] e nas Diretrizes Brasileiras das diversas Sociedades Médicas [2, 3,I4-I7]. As atuações são desenvolvidas por monitores, que são supervisionados por uma equipe multidisciplinar.

\section{PARTICIPANTES}

O público alvo do projeto são os frequentadores adultos dos locais de atuação. O projeto é gratuito e aberto a qualquer pessoa que tenha interesse e disponibilidade para participar de todas ou apenas de parte das atividades. Nos últimos anos, a população atendida foi formada, principalmente, por homens e mulheres com mais de 50 anos e de diferentes classes sociais. Grande parte da população atendida apresenta doenças e/ou fatores de risco cardiovasculares e já pratica algum tipo de atividade física sem orientação em locais públicos.

\section{ATUAÇÕES DE EXTENSÃO}

\section{ATUAÇÃO CONTÍNUA JUNTO AO PARQUE FERNANDO COSTA}

A atuação do Projeto Exercício e Coração no parque Fernando Costa inclui: aulas de alongamento; avaliações físicas; prescrição de exercício; sessões supervisionadas de treinamento; reavaliações; aulas especiais; e eventos pontuais.

As aulas de alongamento envolvem exercícios de alongamento passivo para os principais músculos, visando à preparação musculoesquelética para a atividade física.

As avaliações físicas são compostas por um questionário sobre saúde e atividade física, pela execução de medidas clínicas e por testes físicos. Ao final é solicitada a permissão para a utilização dos dados para pesquisa científica (protocolo aprovado pelo Comitê de Ética da Escola de Educação Física e Esporte da USP). O questionário levanta os dados:

- pessoais (sexo, idade, endereço, escolaridade, entre outros);

- clínicos (sintomas, doenças, fatores de risco e medicamentos);

- de atividade física (se faz, o que faz, quantas vezes por semana e onde).

As medidas realizadas são:

- hemodinâmicas (frequência cardíaca e pressão arterial) [I6];

- metabólicas (glicemia e colesterolemia total de jejum por punção digital) [2,I7];

- antropométricas (massa corporal, estatura e circunferências de cintura e quadril) [3].

Para a avaliação da normalidade das medidas são utilizados os critérios das Diretrizes Médicas Brasileiras [2, 3, I4-I7]. Os testes físicos avaliam a aptidão cardiovascular, força de membros superiores, resistência abdominal, força de membros inferiores, flexibilidade de ombros e flexibilidade lombar, através, respectivamente, dos testes de marcha estacionária [12,I3] ou Cooper [7], flexão de cotovelo [12,I3], abdominais [6], impulsão vertical [I3], flexibilidade de ombros [I2,I3] e sentar e alcançar [6]. A partir dessa avaliação, o risco cardiovascular para a prática do exercício é analisado [I]. Se necessário, a pessoa é encaminhada ao médico, que será responsável por uma avaliação mais detalhada, executando um teste ergométrico e/ou obtendo um atestado para prática não supervisionada.

A prescrição de exercício é feita de forma individualizada visando à saúde geral e, principalmente, a cardiovascular; mas considerando também as 
limitações, os objetivos e a disponibilidade individual. Essa prescrição inclui atividades aeróbicas, que devem ser realizadas pelo menos três vezes por semana, por 30 minutos, em intensidade moderada.

As sessões supervisionadas de treinamento incluem as três primeiras sessões e visam ensinar a execução correta dos exercícios para que a pessoa se torne autônoma.

As reavaliações físicas são idealmente feitas após três a seis meses da avaliação e avaliam a efetividade da prescrição feita. Essa reavaliação consta dos mesmos procedimentos da avaliação e verifica as modificações ocorridas. Se a evolução for adequada, os sujeitos recebem nova prescrição de exercícios aeróbicos e uma orientação para a prática também de exercícios resistidos, que visam uma melhora da saúde musculoesquelética.

As aulas temáticas são oferecidas esporadicamente $e$ constam de atividades lúdicas e diferentes, tendo o objetivo de motivar os participantes e trazer novas experiências.

Os eventos especiais são realizados nos fins de semana e têm o objetivo de incentivar a prática física e apresentar o projeto aos usuários do parque que não o conhecem.

\section{ATUAÇÃO CONTÍNUA JUNTO AO HOSPITAL UNIVERSITÁRIO}

Esta atuação ocorre junto a dois grupos distintos, o de Combate às Doenças Metabólicas e o de Prevenção de Quedas de Idosos. No primeiro grupo, a atuação inclui encontros semestrais com os pacientes, nos quais são ministradas palestras sobre a importância da atividade física e, em seguida, é realizada uma atividade prática para motivação. $\mathrm{Na}$ atuação junto ao grupo de quedas, são atendidos idosos que caíram pelo menos duas vezes no último ano. Esses pacientes fazem uma avaliação física e recebem uma orientação sobre exercícios de alongamento, equilíbrio e fortalecimento muscular, visando reduzir a probabilidade de novas quedas. $\mathrm{O}$ acompanhamento é feito com maior frequência no início, mas as sessões se espaçam com o tempo, tornando o idoso autônomo para esta prática.

\section{ATUAÇÕES PONTUAIS}

Ao longo de sua existência, o projeto atuou em várias campanhas públicas de saúde, estimulando a prática de atividade física, fazendo avaliações de risco cardiovascular e orientando uma prática segura. Essas atuações incluíram palestras, avaliações individualizadas, aulas práticas, entre outras, e contaram com diversos parceiros, como a
Secretaria Municipal do Meio Ambiente, a Sociedade Brasileira de Hipertensão (SBH), a Associação Nacional de Assistência ao Diabético (ANAD), o Serviço Social do Comércio (SESC) e o próprio Hospital Universitário da USP. Atualmente, o projeto desenvolve anualmente atividades no Dia de Prevenção e Combate à Hipertensão Arterial, no Dia de Prevenção e Combate ao Diabetes, no Dia do Desafio e no Dia Mundial da Atividade Física.

\section{ATUAÇÕES DE ENSINO}

Para a execução das atividades do projeto, alunos - principalmente de graduação em Educação Física e Esporte, mas também de pós-graduação - são treinados em relação ao conhecimento sobre os benefícios da atividade física regular para a saúde, sobre como avaliar o risco cardiovascular para a prática, como prescrever uma prática segura, como ministrar essa prática e como avaliar se ela tem os resultados esperados. Esse treinamento é feito de forma teórica, em cursos específicos e em reuniões, mas inclui, principalmente, a atuação prática desses monitores nas atividades do projeto, onde inicialmente observam, depois auxiliam e, finalmente, atuam; sempre com a supervisão próxima de um profissional formado. Assim, o projeto complementa a formação acadêmica do estudante. Esses monitores atuam de forma voluntária ou recebendo bolsas (Bolsa Trabalho da COSEAS, bolsa da Associação de Ambientes e Amigos do Parque da Água Branca - ASSAMAPAB - e, atualmente, bolsas do programa Aprender com Cultura e Extensão).

\section{ATUAÇÕES DE PESQUISA}

Com a finalidade de obter conhecimentos científicos sobre o risco cardiovascular e a prática de atividades físicas em locais públicos, bem como sobre os efeitos do projeto, os dados coletados nas avaliações e reavaliações dos sujeitos que assinaram o termo de consentimento são tabulados e analisados. Essa análise permite a adequação do próprio projeto e gera conhecimento científico sobre a atividade física e a saúde em locais públicos, dando subsídios para outros grupos que pretendam montar programas como esse. Os resultados científicos são publicados na forma de resumos de congressos e artigos completos.

\section{RESULTADOS}

Ao longo desses mais de dez anos de atuação, as atividades do Projeto Exercício e Coração, assim como sua 
possibilidade de atendimento, foram se ampliando. $\mathrm{Ob}-$ viamente, como o projeto ocorre em locais públicos, sua capacidade de atuação oscila em função de questões exteriores ao projeto, como a ocorrência de outros eventos no parque, mudanças climáticas, verbas para campanhas, etc.

Em sua atuação pontual junto ao parque Fernando Costa, nesses mais de dez anos, o projeto realizou mais de I.43I avaliações físicas. Nos últimos quatro anos (2007 a 2010), após sua ampliação, a média foi de I5O avaliações por ano. As reavaliações começaram em 2002 e foram realizadas mais de 4I4, sendo a média de 2007 a 2010 de 59 reavaliações anuais (ver Anexo, Figura I).

As aulas de alongamento se iniciaram em 2004. No início eram ministradas apenas duas aulas semanais, mas desde 2006 esse número foi ampliado para nove aulas por semana, totalizando nos últimos anos cerca de 390 aulas por ano e I4.263 atendimentos anuais (ver Anexo, Figura 2).

Considerando-se as sessões de supervisão de treinamento, elas passaram a ser executadas no projeto a partir de 2006 e, a partir daí, já foram realizadas I.8I8 sessões, numa média de 355 sessões por ano nos últimos quatro anos - 2007 a 2010 (ver Anexo, Figura 3).

$\mathrm{Na}$ atuação junto ao HU-USP iniciou em 2008 e, nesse período, o projeto realizou I89 atendimentos, sendo 45 junto ao Grupo de Quedas e o restante no de Doenças Metabólicas.

Considerando-se a participação em campanhas e eventos públicos de saúde, o projeto participou ao longo de sua existência de 28 eventos, atendendo mais de I0.382 pessoas (ver Anexo, Figura 4).

Em relação à sua vertente de ensino, 47 alunos de graduação foram monitores no parque Fernando Costa, com uma oscilação de 2 a 4 monitores bolsistas por ano. Além disso, nas atividades pontuais em campanhas públicas, uma média de IO a I5 monitores atuam por evento, de modo que ao longo dos anos, mais de I50 alunos foram treinados nessa atuação.

Considerando-se a produção científica, os dados do Projeto deram origem a II monografias de graduação, 40 resumos apresentados em congressos científicos e II artigos completos publicados em revistas científicas.

\section{DISCUSSÃO E CONCLUSÕES}

Diante do exposto, observa-se que embora o enfoque principal do Projeto Exercício e Coração seja a assistência à população, ele também atua em duas outras vertentes relacionadas à formação profissional e à pesquisa científica.

A respeito da assistência à comunidade, o principal elemento do projeto, este tem atingido seus objetivos com excelência, atendendo um grande número de pessoas por ano em suas várias atuações. De fato, a grande gama de atuações do projeto permite atingir o objetivo de incentivar a prática segura de atividades físicas visando à saúde cardiovascular em diferentes níveis, desde pessoas que vão a campanhas públicas e ainda não têm consciência da necessidade de atividade física para sua saúde - incluindo pessoas que reconhecem essa importância, mas ainda não sabem o que fazer - até aquelas que já praticam atividades físicas, mas precisam de uma orientação para maximizar os benefícios dessa prática.

Considerando-se a formação profissional, o projeto também tem atingindo seus objetivos, ajudando na formação de profissionais mais competentes e com uma experiência prática rica e única para a atuação na prevenção e reabilitação de doenças crônico-degenerativas.

Do ponto de vista científico, o projeto caracteriza-se como um dos poucos projetos de pesquisa que avalia os efeitos e as limitações da prática de atividade física para a saúde em condições reais, ou seja, sem o controle excessivo de variáveis intervenientes, o que faz com que os dados obtidos com essa pesquisa tenham grande validade externa e, portanto, sirvam de embasamento e, talvez, modelo, para a elaboração de outras propostas de intervenção pública no mesmo sentido.

Assim, o Projeto Exercício e Coração atua fortemente como um projeto de extensão dentro de uma universidade pública, preocupando-se também com o ensino e a pesquisa.

\section{REFERÊNCIAS}

[I] AMERICAN GOLLEGE OF SPORTS MEDIGINE. ACSM's guidelines for exercise testing and prescription. Philadelphia: Lippincott Williams \& Wilkins, 2000.

[2] AMERICAN DIABETES ASSOGIATION. Diagnosis and classification of diabetes Mellitus. Diabetes Care, v. 27, Supplement I, S5-Sio, 2004.

[3] ASSOCIAÇÃO BRASILEIRA PARA O ESTUDO DA OBESIDADE E DA SÍNDROME METABÓLICA. 
Diretrizes brasileiras de obesidade 2009-2010. 3 . ed. Itapevi: AG Farmacêutica, 2009.

[4] BRASIL. Ministério da Saúde. Secretaria de Vigilância em Saúde. Departamento de Análise de Situação de Saúde. Plano de ações estratégicas para o enfrentamento das doenças crônicas não transmissíveis (DCNT) no Brasil 20II-2022. Brasília: Ministério da Saúde, 20II.

[5] BRASIL. Ministério da Saúde. Secretaria de Vigilância em Saúde. Secretaria de Gestão Estratégica e Participativa. Vigitel Brasil 2009: vigilância de fatores de risco e proteção para doenças crônicas por inquérito telefônico. Brasília: Ministério da Saúde, 2010.

[6] GANADIAN SOGIETY FOR EXERGISE PHYSIOLOGY. Canadian Physical Activity, Fitness \& Lifestyle Approach: CSEP-Health \& Fitness Program's Health-Related Appraisal and Counseling Strategy, 3. ed. Ottawa: CSEP, 2003.

[7] GOOPER, K. H. Capacidade Aeróbica. Rio de Janeiro: Forum. I972.

[8] FORJAZ, G. L. et al. Assessment of the cardiovascular risk and physical activity of individuals exercising at a public park in the city of São Paulo. Arquivos Brasileiros de Cardiologia, v. 79, n. I, p. 43-50, 2002.

[9] FORJAZ, C. L. et al. Projeto Exercício e Coração. In: UNIVERSIDADE DE SÃO PAULO. Escola de Educação Física e Esporte. Memória dos cursos comunitários e de extensão da EEFE-USP. São Paulo: Escola de Educação Física e Esporte da USP, 2009, p.I23-I48.

[IO] MATSUDO, V. et al. Promotion of physical activity in a developing country: the Agita São Paulo experience. Public Health Nutr., v. 5, Ia, p. 253-26I, 2002.

[iI] MATSUDO, V. et al. Time Trends in Physical Activity in the State of São Paulo, Brazil: 2002-2008. Medicine and Science in Sports and Exercise, v. 42, n. I2, p. 223I-2236, 2010.

[I2] RILKI, R. E.; JONES, G. J. Development and validation of a functional fitness test for communityresiding older adults. Journal of Aging and Physical Activity, v. 7, n. 2, p. I29-6I, I999.

[I3] RILKI, R. E.; JONES, C. J. Functional fitness normative scores for community-residing older adults, ages 60-94. Journal of Aging and Physical Activity, v. 7, n. 2, p. I62-8I, I999.

[I4] SOGIEDADE BRASILEIRA DE GARDIOLOGIA. III diretrizes brasileiras sobre dislipidemias e diretriz de prevenção da aterosclerose do Departamento de Aterosclerose da Sociedade Brasileira de
Cardiologia. Arq. Bras. Cardiol., v. 77, suppl. 3, p. I-48, $200 \mathrm{I}$.

[I5] SOGIEDADE BRASILEIRA DE GARDIOLOGIA. I Brazilian guidelines on diagnosis and treatment of metabolic syndrome. Arq. Bras. Cardiol., v. 84, suppl. I, p. I-28, 2005.

[i6] SOCIEDADE BRASILEIRA DE HIPERTENSÃO. VI diretrizes brasileiras de hipertensão arterial. Revista Brasileira de Hipertensão, vol. I7, n. I, p. I-64, 20 IO.

[I7] SPOSITO A. C. et al. IV Brazilian guideline for dyslipidemia and atherosclerosis prevention: $\mathrm{De}$ partment of Atherosclerosis of Brazilian Society of Cardiology. Arq. Bras. Cardiol., vol. 88, suppl. I, p. 2-I9, 2007.

[I8] WORLD HEALTH ORGANIZATION. Global recommendations on physical activity for health. Genebra: World Health Organization, 2010.

[ig] WORLD HEALTH ORGANIZATION. World Health Day 2002. Sedentary lifestyle: a global health problem. Genebra: World Health Organization, 2002. Disponível em: <http://www.who.int/world-heathyday $>$. Atualizado em 2002, consultado em 2008.

\section{AGRADECIMENTOS}

O projeto contou ao longo dos anos com vários parceiros: administração do Parque Fernando Costa, ASSAMAPAB, Gentro de Atendimento Psicossocial de Perdizes (CAPS-Perdizes), Secretaria do Meio Ambiente da Prefeitura de São Paulo, Coordenadoria de Comunicação Social da USP (CGS-USP), Empresa Junior da EEFE-USP, COSEAS-USP, Pró-Reitoria de Cultura e Extensão da USP, SBH, ANAD, HUUSP, SESG e Fundo Social de Solidariedade do Estado de São Paulo. Além disso, o projeto agradece aos seus monitores, coordenadores e usuários. 


\section{ANEXO}

\section{FIGURA 1}

Painel A

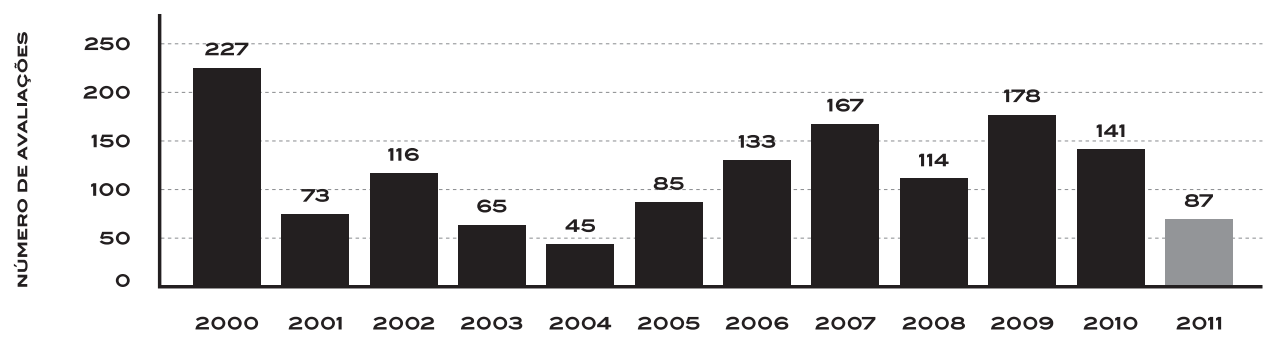

Painel B

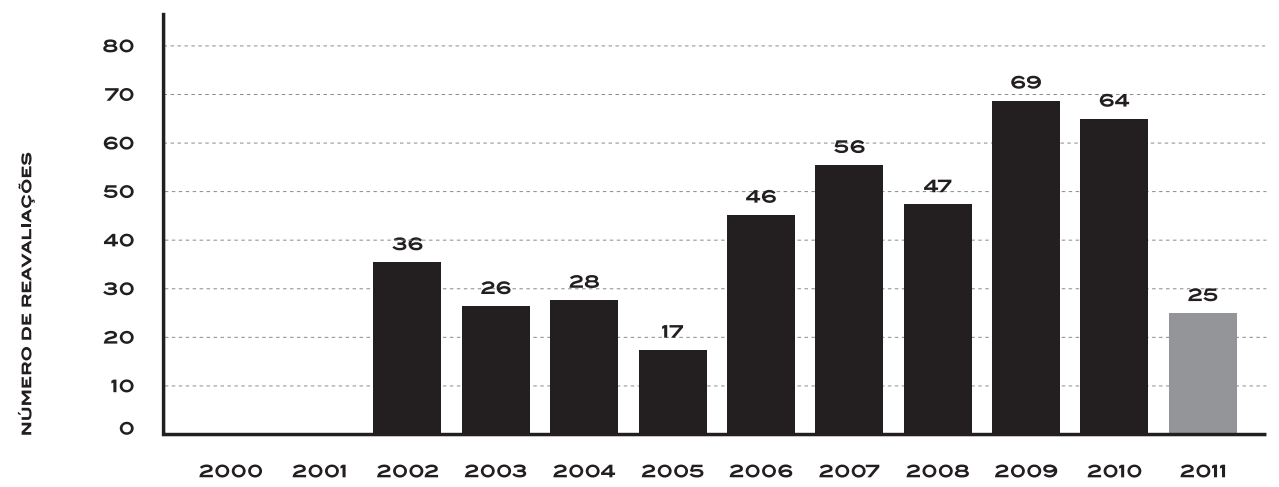

Número de avaliações (painel A) e reavaliações (painel B) realizadas pelo Projeto Exercício e Coração no parque Fernando Ciosta de 2000 a 20II. Nota: os dados de $201 \mathrm{I}$ referem-se ao período de janeiro a junho. 
FIGURA 2

Painel A

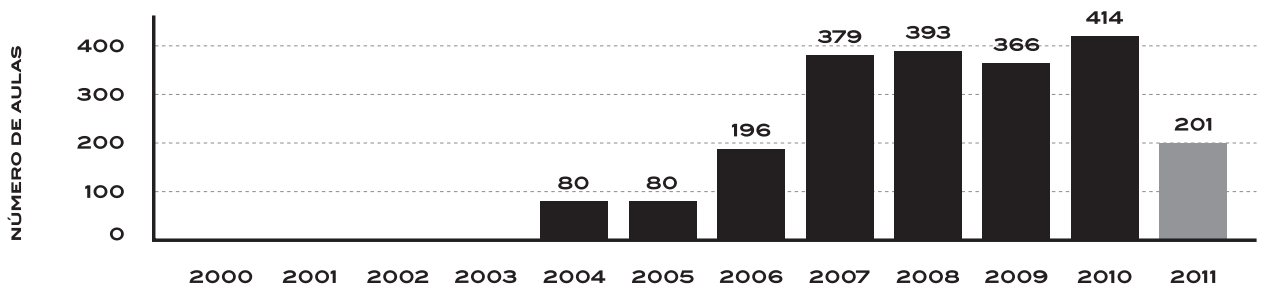

\section{Painel B}

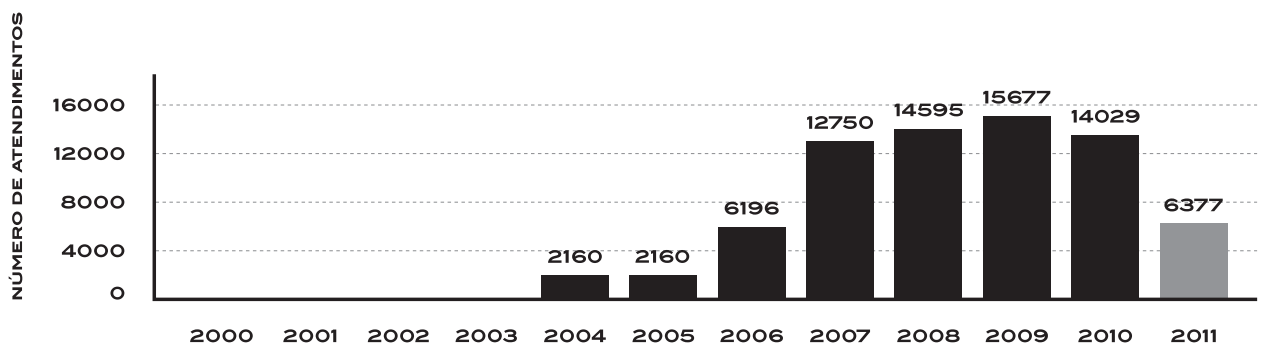

Número de aulas (painel A) e de atendimentos (painel B) de alongamento realizados no parque Fernando Costa pelo Projeto Exercício e Coração de 2000 a 20II. Nota: os dados de 2011 referem-se ao período de janeiro a junho.

\section{FIGURA 3}

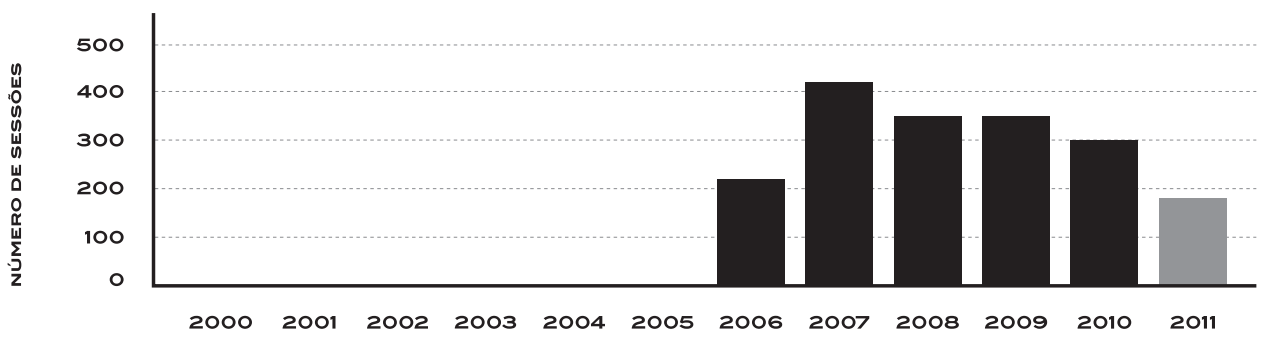

Número de sessões de supervisão de treinamento realizadas por ano pelo Projeto Exercício e Coração no parque Fernando Costa de 2006 a 20II. Nota: os dados de $201 \mathrm{I}$ referem-se ao período de janeiro a junho. 
FIGURA 4

Painel A

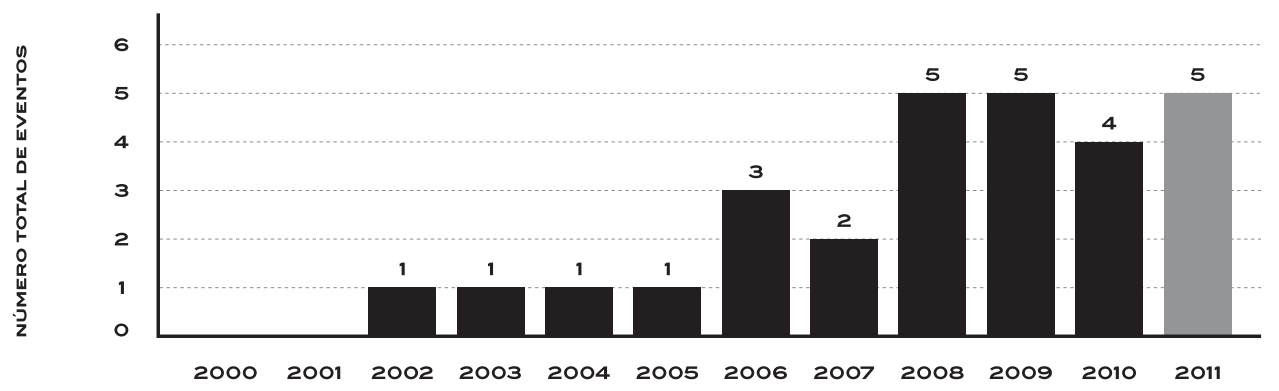

Painel B

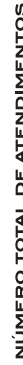

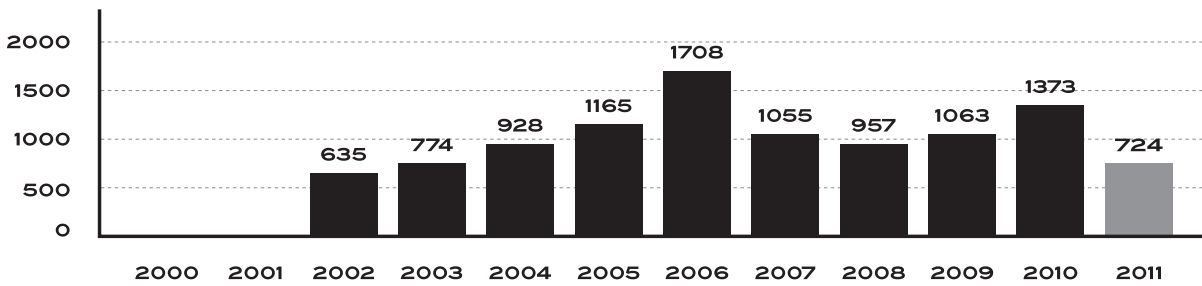

Número total de eventos de saúde (painel A) e de atendimentos nesses eventos (painel B) realizados pelo Projeto Exercício e Coração de 2003 a 20II. Nota: os dados de 2011 referem-se ao período de janeiro a junho. 